\title{
Corn Seed Defect Detection Based on Watershed Algorithm and Two-Pathway Convolutional Neural Networks
}

\author{
Linbai Wang ${ }^{1,2+}$, Jingyan Liu ${ }^{1,2+}$, Jun Zhang ${ }^{1,2}$, Jing Wang ${ }^{1,2}$ and Xiaofei Fan ${ }^{1,2 *}$ \\ ${ }^{1}$ State Key Laboratory of North China Crop Improvement and Regulation, Hebei Agricultural University, Baoding, China, \\ ${ }^{2}$ College of Mechanical and Electrical Engineering, Hebei Agricultural University, Baoding, China
}

OPEN ACCESS

Edited by:

Lisbeth Garbrecht Thygesen,

University of Copenhagen, Denmark

Reviewed by:

Abhimanyu Singh Garhwal,

AgResearch Ltd., New Zealand

Lin Qi,

Ocean University of China, China

${ }^{*}$ Correspondence:

Xiaofei Fan

leopardfxf@163.com

${ }^{t}$ These authors have contributed equally to this work and share first

authorship

Specialty section:

This article was submitted to Technical Advances in Plant Science, a section of the journal

Frontiers in Plant Science

Received: 24 June 2021

Accepted: 25 January 2022

Published: 23 February 2022

Citation:

Wang L, Liu J, Zhang J, Wang J and Fan X (2022) Corn Seed Defect

Detection Based on Watershed Algorithm and Two-Pathway

Convolutional Neural Networks.

Front. Plant Sci. 13:730190.

doi: 10.3389/fp/s.2022.730190
Corn seed materials of different quality were imaged, and a method for defect detection was developed based on a watershed algorithm combined with a two-pathway convolutional neural network (CNN) model. In this study, RGB and near-infrared (NIR) images were acquired with a multispectral camera to train the model, which was proved to be effective in identifying defective seeds and defect-free seeds, with an averaged accuracy of $95.63 \%$, an averaged recall rate of $95.29 \%$, and an F1 (harmonic average evaluation) of $95.46 \%$. Our proposed method was superior to the traditional method that employs a one-pathway CNN with 3-channel RGB images. At the same time, the influence of different parameter settings on the model training was studied. Finally, the application of the object detection method in corn seed defect detection, which may provide an effective tool for high-throughput quality control of corn seeds, was discussed.

Keywords: corn seed defect, multispectral image, object detection, watershed segmentation algorithm, convolutional neural network

\section{INTRODUCTION}

Corn is one of the most important crops in the world (Afzal et al., 2017), which is widely planted around the Earth. Its output and trade volume have kept increasing in recent years. In the process of circulation, appearance quality is a critical factor that influences corn seed price. Corn seeds are vulnerable to damage and mildew during storage and transportation, and phenotypic defect is an important index of seed quality evaluation. At present, seed quality detection still relies on the method of traditional manual identification, which employs low efficiency and strong subjectivity. With the development of computer vision technology (Rehman et al., 2018; Gutiérrez et al., 2019; Keiichi et al., 2019; Azimi et al., 2020; Arunachalam and Andreasson, 2021), image processing methods based on machine learning are applied to seed quality classification and have achieved good results. Kiratiratanapruk and Sinthupinyo (2012) proposed a method to classify more than 10 levels of seed quality by using color and texture features with a support vector machine (SVM) classifier. Ke-Ling et al. (2018) proposed a method of high-quality pepper seed screening based on machine vision, which could be used to predict the germination rate of seeds effectively, and therefore provided a guide for seed quality selection. Ali et al. (2020) discussed the feasibility of the machine learning method in corn seed classification. While the traditional machine learning methods normally require extracting the features manually, which are usually not comprehensive enough, the recognition accuracy, therefore, is limited. 
In recent years, as a representative of deep learning technology, convolutional neural networks (CNNs) develop rapidly and are widely used for image recognition (Afonso et al., 2019; Altuntaş et al., 2019; Gao et al., 2020; Zhang C. et al., 2020). Compared with traditional machine learning technology, CNNs are naturally embedded with a feature learning part through the combination of low-level features to form more abstract highlevel features. Many researchers have applied CNNs to the field of agriculture. Laabassi et al. (2021) proposed a CNN model to classify wheat varieties, and the accuracy of classification was between 85.00 and $95.68 \%$. Pang et al. (2020) developed a method for rapid estimation and prediction of corn seed vigor using a hyperspectral imaging system with deep learning. The recognition accuracy of the $1 \mathrm{D}-\mathrm{CNN}$ model reached $90.11 \%$, and the recognition accuracy of the 2D-CNN model reached $99.96 \%$. $\mathrm{Sj}$ et al. (2021) proposed a method to extract the characteristics of corn seeds by using a deep CNN and then classifying the varieties. The results showed that CNNs were effective in corn seed classification.

In this article, RGB and NIR images (Kusumaningrum et al., 2018) collected by a multispectral camera were used to train a CNN model. To solve the problem of corn seed adhesion and seed location during the recognition process, a watershed algorithm (Lei et al., 2019; Sta et al., 2019; Zhang et al., 2021) combined with a two-way CNN (Zhang J. J. et al., 2020) was proposed to detect corn seed defects. The results revealed that this method is with high accuracy, and the targets can be accurately located and classified. This method may provide a theoretical basis for the subsequent development of a seed quality control device.

\section{MATERIALS AND METHODS}

\section{Experimental Material and Instruments}

In this experiment, 2,365 corn seeds from three different varieties (Zhengdan 985, Keshi 982, Jiyu 517) were adopted as experimental materials. Some seeds were defect-free in appearance, and the other seeds were with defects, including mold, insect or mechanical damages, and discoloration. A 4channel (RGB + NIR) multispectral camera (LQ-200CL, JAI, Denmark) was used for image acquisition, with 8 bits for each channel and a resolution of 1,296* 964. A white LED ring light, coupled with a near-infrared ring light, and a white backlight panel were used to enhance the image contrast. The image acquisition platform is shown in Figure 1. At the same time, to prevent the seeds from overlapping, the vibration module was placed under the backlight panel (shown in Figure 2). The motor voltage is $12 \mathrm{~V}$. The rotational speed of the motor is $8,000 \mathrm{rpm}$. The size of the vibrating head is $3.5 \mathrm{~cm}$. It is found that the vibration module shows a very good effect in restraining seed overlap and shielding.

The experiment was based on Windows 10, a 64-bit operating system with CUDA 10.0, and python programming language, along with TensorFlow and Keras deep learning framework. The computer used for the experiment employed a GeForce GTX 1660 graphics card, with 6G memory, and an Intel (R) Core (TM) i5-9400f processor with the main frequency of $2.90 \mathrm{GHz}$.

\section{Data Acquisition}

A total of 50 samples of corn seed with no defects (1,066 single seeds overall) and fifty seed samples with different appearance defects (1,042 single seeds overall) were imaged. The images of another 10 samples with both defective and defect-free seeds were also acquired for the verification of the final model, with an overall 257 single seeds. Each sample was captured in one image deck, which contained RGB and NIR images, with a size of 1,296 × 964. The images acquired are shown in Figure 3 . To solve the issue of adhesion among seeds in the images, a watershed algorithm was applied to each image, and all individual seeds were segmented. Eventually, each seed was extracted from the original image to form a new image, which was resized to $224 \times 224$ with bilinear interpolation.

To improve the performance of the model, data augmentation was implemented for image decks of individual seeds. The enhancement methods (Huang et al., 2019; Tiwari et al., 2021) included brightness adjustment, rotation, applying Gaussian noise, etc. The images of defect-free seeds were labeled as "good," and the images of defective seeds were labeled as "bad." Eventually, there were 3,913 images (RGB + NIR) of defectfree seeds and 3,913 images of defective seeds, respectively. The training set and the testing set were divided by $4: 1$, and therefore, 5,869 images with single seed were used for training, and 1,957 images were used for testing.

\section{Watershed Algorithm}

Every single seed in the image deck was segmented using the watershed algorithm. First, the original 4-channel image was converted to a grayscale image. By comparing the four layers ( $R, G, B$, and NIR), the results showed that the B-channel image was the best to use for binarization. Binarization was then performed, and any noise in the binary image was removed by a morphological open operation. An expansion operation was then applied to the binary image, and a distance transforming algorithm was used to obtain the central region of each seed. The edge of the seed was the dilational image subtracted from the central regions. The central region of each seed was then naturally separated from each other. Finally, the watershed algorithm was used to extract the edge of the seeds, and each seed was segmented in the image by position coordinates. The segmentation processes are shown in Figure 4. The NIR images were then segmented using the position coordinates from the segmented RGB images. The combination of the RGB image and NIR image of each seed was used for training or detection processes.

\section{Corn-Seed-Net Model Structure}

Every single seed was separated by the watershed algorithm, and the position coordinates were obtained. The CNN model was then used to detect the quality of the corn seeds. The detection results were marked in the image according to the position coordinates. In this article, a two-pathway CNN, Corn-seed-Net (shown as Figure 5), was designed combining VGG16 (Simonyan and Zisserman, 2015) and ResNet50 (He et al., 2016). The model was used to extract deep features of 4-channel corn seed images and then classify them. 


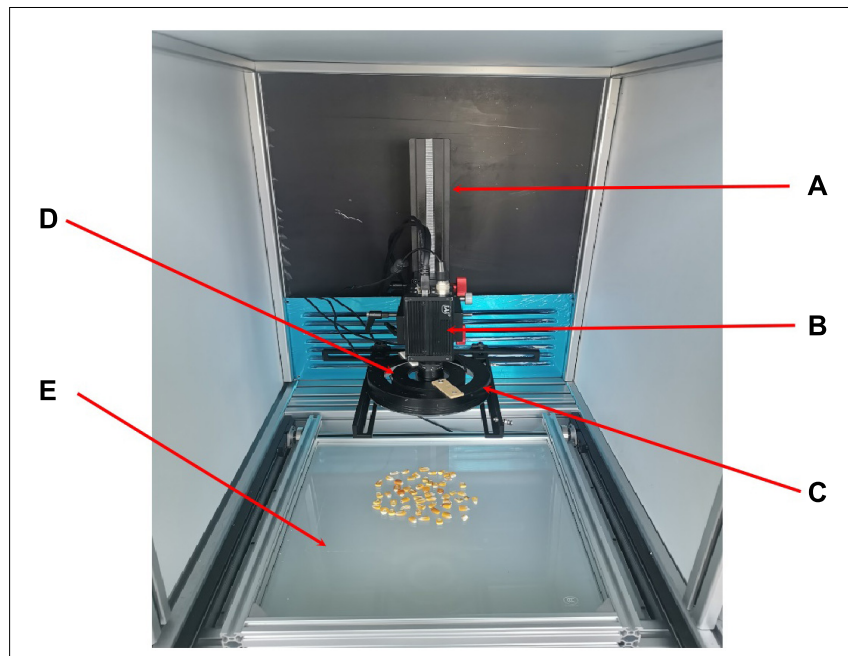

FIGURE 1 | Image acquisition platform. (A) Support, (B) camera, (C) circular white light source, (D) circular near-infrared light source, and (E) white backlight.

To reduce the number of parameters, continuous convolution kernels of $3 \times 3$ were used in the VGG16. Thirteen convolution layers were used to extract deeper image features and increase the fitting capacity and the expressive capacity of the model. However, as the number of network layers increases, the gradient of the model disappears or explodes, which makes the performance of the model plummet. However, the residual structure was added in the ResNet50, the input of the convolution layer was directly added to the output of the convolution layer, and it solves the degradation problem of deep CNN. Therefore, the advantages of both VGG16 and ResNet50 were combined in the Corn-seed-Net.

In this article, the VGG16 branch was optimized. The number of parameters of the last two fully connected layers of the original models was tremendous. To avoid feature information redundancy, a convolution layer of $7 \times 7$ was applied to the final max-pooling layer, with 512 channels, and two fully connected layers composed of 512 feature vectors were added. In this way, the number of parameters was reduced. For the ResNet50 branch, after the global average pooling layer, a fully connected layer composed of 512 feature vectors was added. The two branches were then fused with the final fully connected layer, and the vectors of the generated features were 1,024. Finally, the classification was completed through the Softmax layer, with the category number set to 2 .

The Softmax function was used to calculate the probability of classification, and the calculation formula is as follows:

$$
y_{i m}=\frac{e^{z_{i m}}}{\sum_{k=1}^{k} e^{z_{i k}}}
$$

In the formula, $y_{i m}$ is the prediction probability that the $i$ th sample belongs to class $m, k$ is the number of categories, $z_{i m}$ is the product of the output vector of the $i$ th sample and the parameter

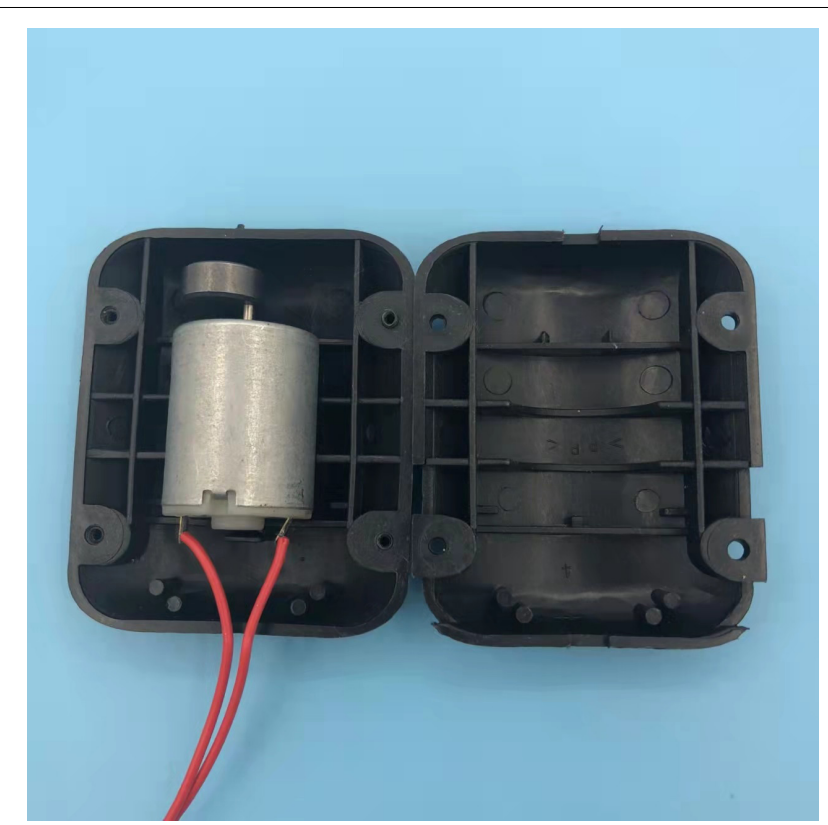

FIGURE 2 | The vibration module.

vector of class $m$, and $z_{i k}$ is the product of the output vector of the $i$ th sample and the parameter vector of class $k$.

Categorical cross-entropy was used to calculate the loss function of the model, and the formula is given as follows:

$$
L=-\sum_{i=1}^{n} \hat{y}_{i m} \lg y_{i m}
$$

In the formula, $L$ is the loss function, $n$ is the number of images in each batch, and $y_{i m}$ is the expected probability that the $i$ th sample belongs to class $m$.

\section{Parameter Set}

To achieve the best result, the model was trained by setting different parameters. The momentum used in the final model was 0.9 , and the initial value of the learning rate was set as 0.001. Stochastic gradient descent (SGD) (Samik and Sukhendu, 2018) algorithm was used with 100 epochs for the training. In the process of training, when the loss of the test set no longer decreased, the learning rate was reduced by half. Other parameters were set to default. The accuracy of the final training set was $100.00 \%$, and the accuracy of the test set was $96.90 \%$.

\section{Hand-Crafted Feature Extraction}

In this article, five hand-crafted feature extraction methods were used to extract the features of a single seed segmented by watershed algorithm, and then an SVM classifier was used for seed classification. The feature extraction methods were as follows:

(1) Morphological characteristics (MC) (Zhang L. et al., 2020) were used to binarize each seed's image. The ratio of the perimeter of the seed area, the diameter of the circle with 

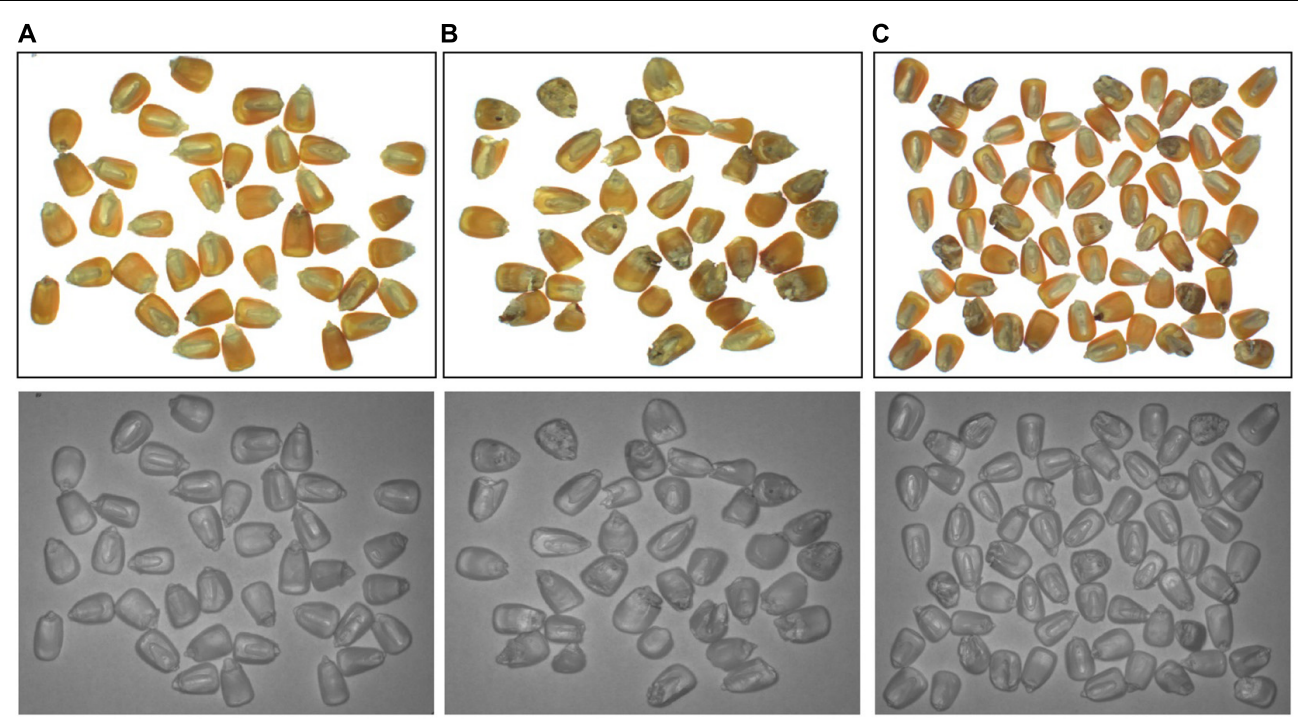

FIGURE 3 | The original image of (A) good quality corn seeds, (B) disfigured corn seeds, and (C) both situations.

A
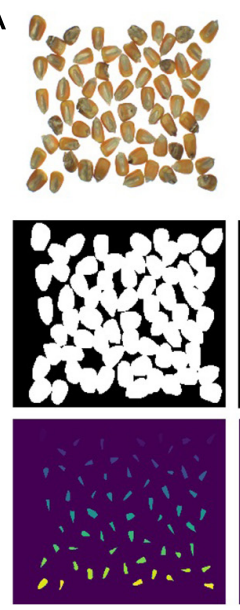
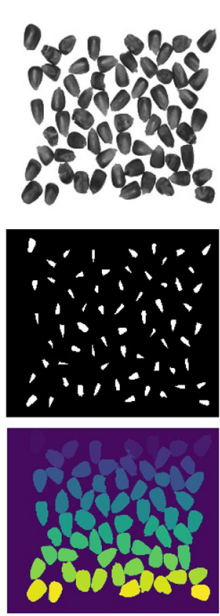
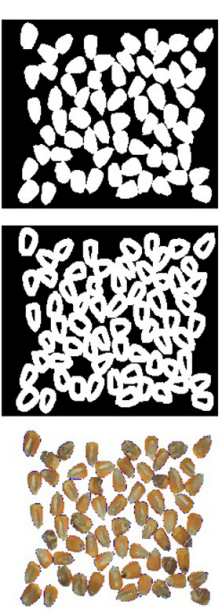

B
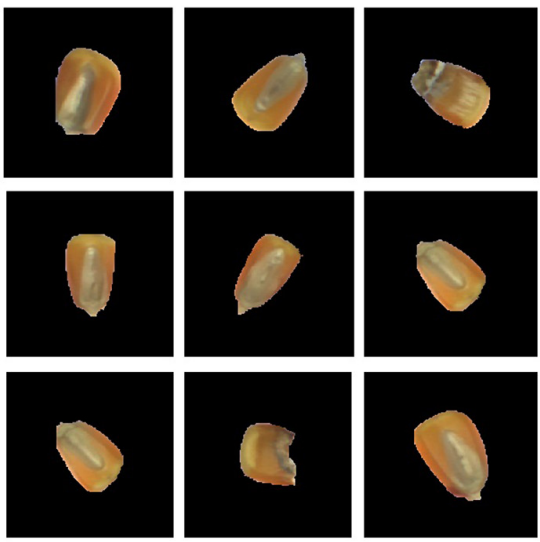

FIGURE 4 | Image processing procedures. (A) Segmentation processes and (B) segmentation results.

the same area, the eccentricity of the fitted ellipse, the ratio of the major axis to minor axis, and the ratio of area to bounding box area from a connected domain were then extracted. A total of five morphological features were used as feature vectors.

(2) Color features have little dependence on image size and position. In this article, the parameters related to color (RGB) histogram were extracted as feature vectors.

(3) Local shape information can be well captured by histogram of gradient (HOG) (Dalal, 2005), and it is relatively stable to the change of geometry and optics. In this article, the gradient information of the image was extracted as feature vectors.

(4) Gray-level co-occurrence matrix (GLCM) (Haralick et al., 1973 ) is a method of texture feature extraction based on statistics. The statistics constructed in this article include contrast, dissimilarity, homogeneity, energy, correlation, and angular second moment. These six characteristic parameters were used as feature vectors.

(5) Local binary pattern (LBP) (Ojala et al., 2002) features have the advantages of gray invariance and rotation invariant. In this article, the LBP value of the image was extracted and used to represent the texture information of the region. Finally, the statistical histogram of LBP features was used as the feature vectors.

\section{Evaluation Index}

In this article, to evaluate the accuracy and stability of the training model for seed quality identification, the precision and recall ratios were used to evaluate the model, and the $F_{1}$ value was used 

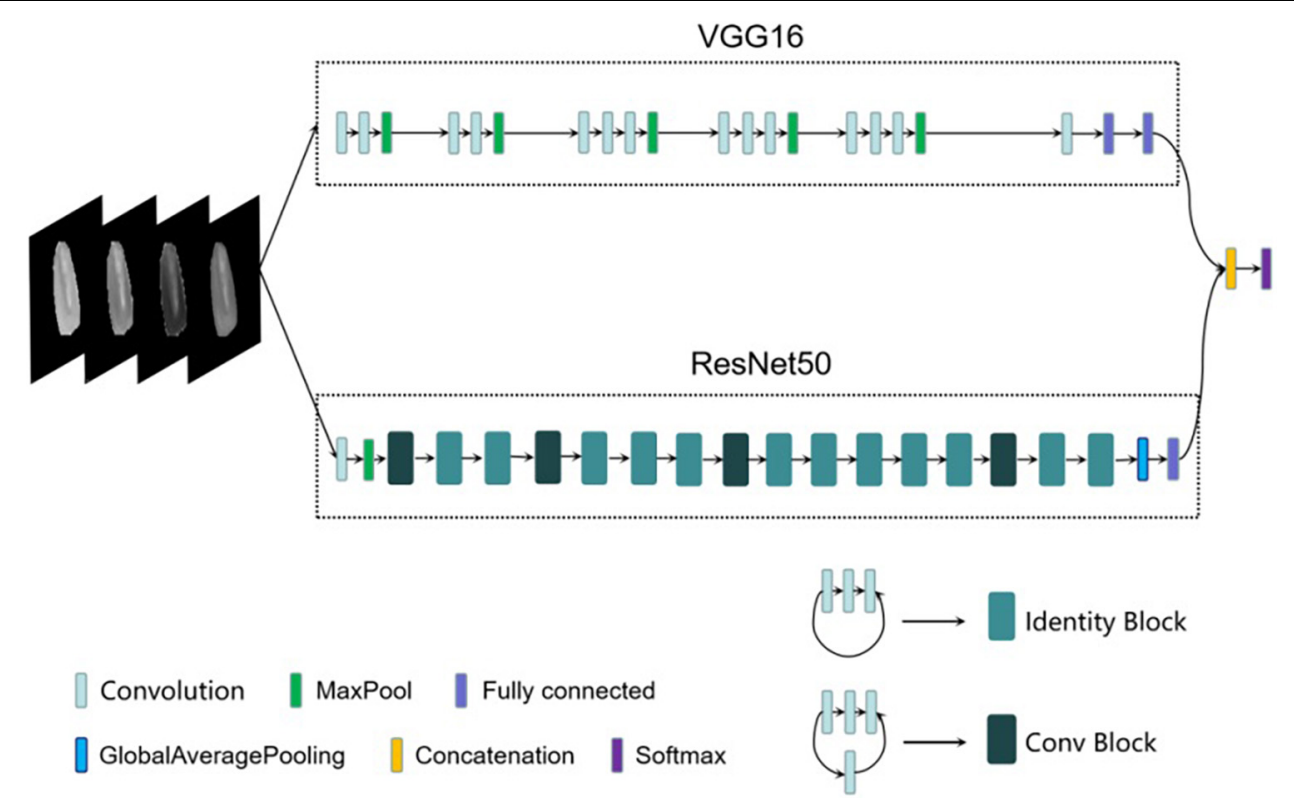

FIGURE 5 | Corn-seed-Net network architecture.

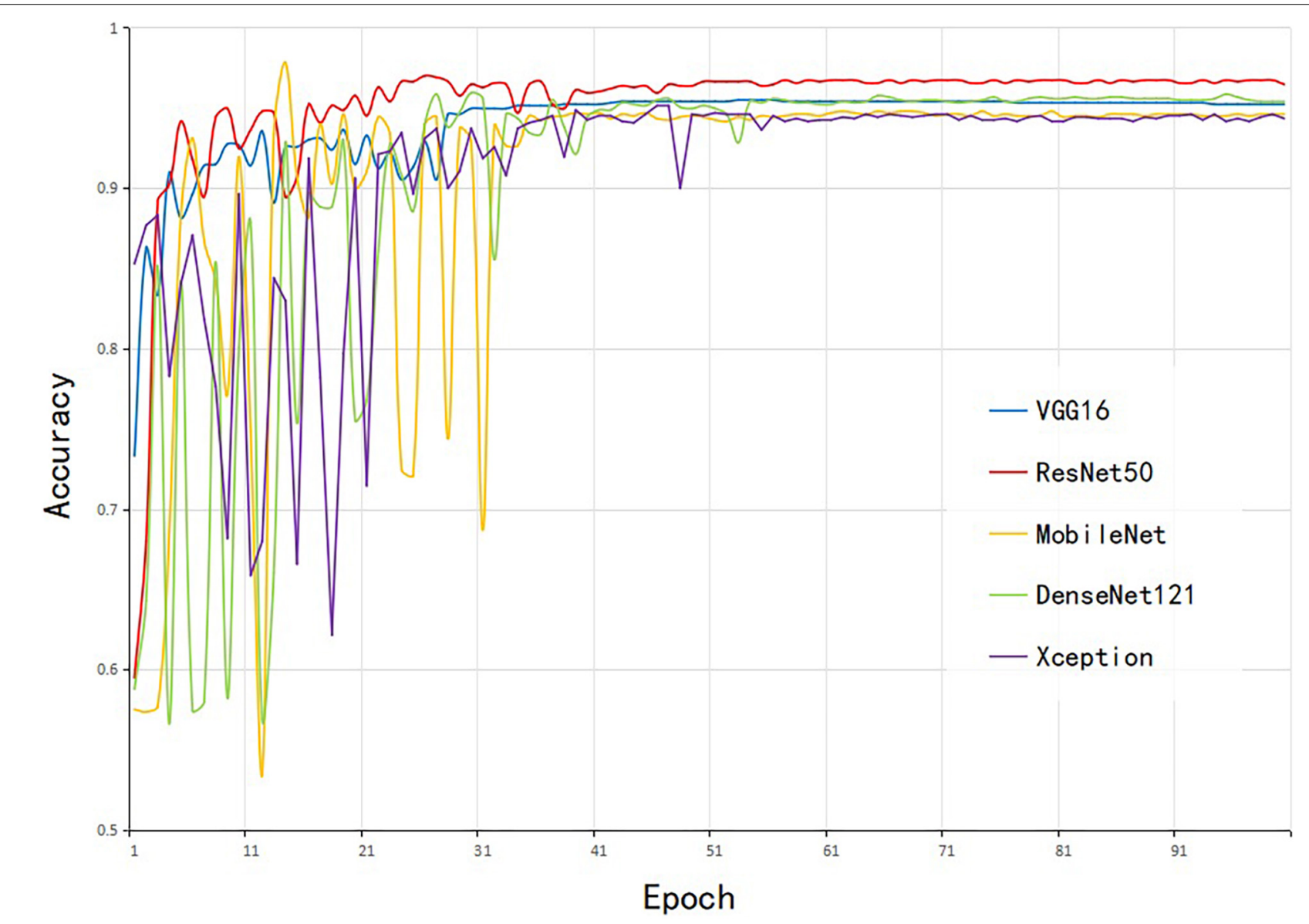

FIGURE 6 | The accuracy of the five models for the test set. 
TABLE 1 | Training results of Corn-seed-Net with different model parameters.

\begin{tabular}{lllll}
\hline $\begin{array}{l}\text { Initial learning } \\
\text { rate }\end{array}$ & $\begin{array}{l}\text { Training } \\
\text { algorithm }\end{array}$ & $\begin{array}{l}\text { Epoch } \\
\text { time/s }\end{array}$ & $\begin{array}{l}\text { Training } \\
\text { accuracy/\% }\end{array}$ & $\begin{array}{l}\text { Validation } \\
\text { accuracy/\% }\end{array}$ \\
\hline 0.001 & Adam & 180 & 100.00 & 94.23 \\
0.0001 & Adam & 180 & 100.00 & 95.80 \\
0.001 & SGD & 165 & 100.00 & 96.90 \\
0.0001 & SGD & 165 & 99.98 & 94.59 \\
\hline
\end{tabular}

as the average evaluation of them. The evaluation formulas are given as follows:

$$
\begin{gathered}
p=\frac{n \mathrm{TP}}{n \mathrm{TP}+n \mathrm{TF}} \times 100 \% \\
R=\frac{n \mathrm{TP}}{n \mathrm{TP}+n \mathrm{FN}} \times 100 \% \\
F 1=\frac{2 P R}{P+R} \times 100 \%
\end{gathered}
$$

where $n_{T P}$ is the number of corn seeds correctly identified, $n_{F P}$ is the number of misidentified corn seeds, and $n_{F N}$ is the number of unrecognized corn seeds.
RESULTS AND DISCUSSION

\section{The Selection of Corn-Seed-Net Model Structure}

To select an optimal model structure, the 4-channel images (RGB + NIR) were used for training five CNN models [e.g., VGG16, ResNet50, MobileNet, DenseNet121 (Huang et al., 2016), and Xception (Chollet, 2017)]. The weights trained on the ImageNet dataset were used for parameter initialization, and the same dataset was trained for the model; the accuracy of the test set is shown in Figure 6. The number of input channels for the model was set to 4 , but the number of convolution layers was unchanged. The number of parameters for convolutional layers was the same as the original model, and therefore, the pre-trained weights from ImageNet were used in this study. The five models converged after 50 epochs, and the accuracy stabilized at a high value. For the five models, the ResNet50 model had the highest accuracy of $96.63 \%$. The VGG16 model converged most rapidly. The DenseNet model achieved the characteristics of dense connection through repeated splicing, but the running memory consumption was large and the convergence time was long. The MobileNet model possessed a smaller amount of parameters but the accuracy was low. Deep separable convolution and residual connection were

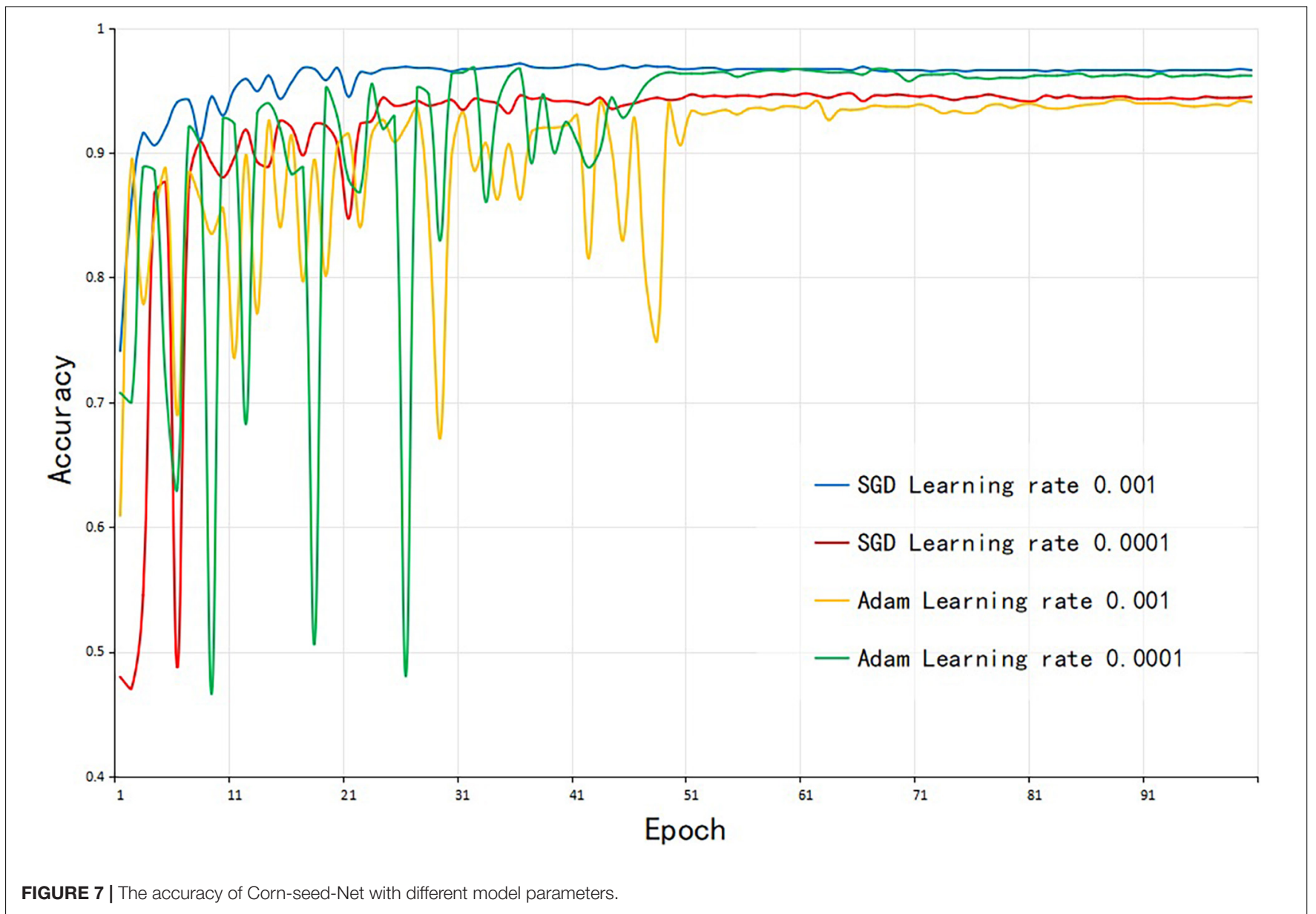


TABLE 2 | Test results of single seed with different models.

\begin{tabular}{|c|c|c|c|c|c|c|}
\hline \multirow[t]{2}{*}{ Model } & \multirow[t]{2}{*}{ Classes } & \multicolumn{2}{|c|}{ Predict classes } & \multicolumn{3}{|c|}{ Model performance } \\
\hline & & Good & Bad & Accuracy $/ \%$ & Averaged accuracy $/ \%$ & Detection time/ms \\
\hline \multirow[t]{2}{*}{ VGG16 } & Good & 99 & 1 & 99 & 99.00 & 45.5 \\
\hline & Bad & 1 & 99 & 99 & & \\
\hline \multirow[t]{2}{*}{ ResNet50 } & Good & 100 & 0 & 100 & 99.00 & 41.7 \\
\hline & Bad & 2 & 98 & 98 & & \\
\hline \multirow[t]{2}{*}{ MobeliNet } & Good & 97 & 3 & 97 & 98.00 & 22.9 \\
\hline & Bad & 1 & 99 & 99 & & \\
\hline \multirow[t]{2}{*}{ DenseNet121 } & Good & 97 & 3 & 97 & 97.50 & 58.0 \\
\hline & Bad & 2 & 98 & 98 & & \\
\hline \multirow[t]{2}{*}{ Xception } & Good & 98 & 2 & 98 & 97.50 & 41.0 \\
\hline & Bad & 3 & 97 & 97 & & \\
\hline \multirow[t]{2}{*}{ Corn-seed-Net } & Good & 100 & 0 & 100 & 100.00 & 68.0 \\
\hline & Bad & 0 & 100 & 100 & & \\
\hline
\end{tabular}
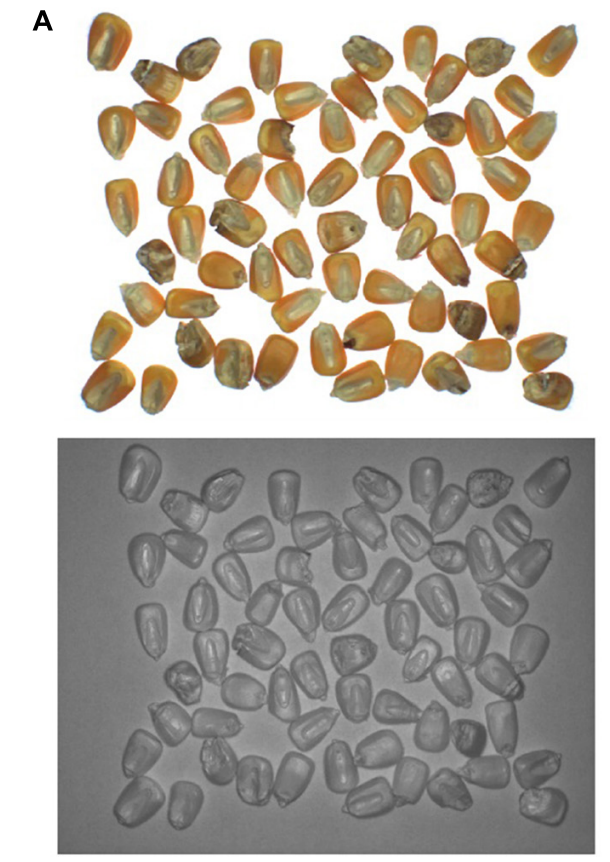

B good 1.0

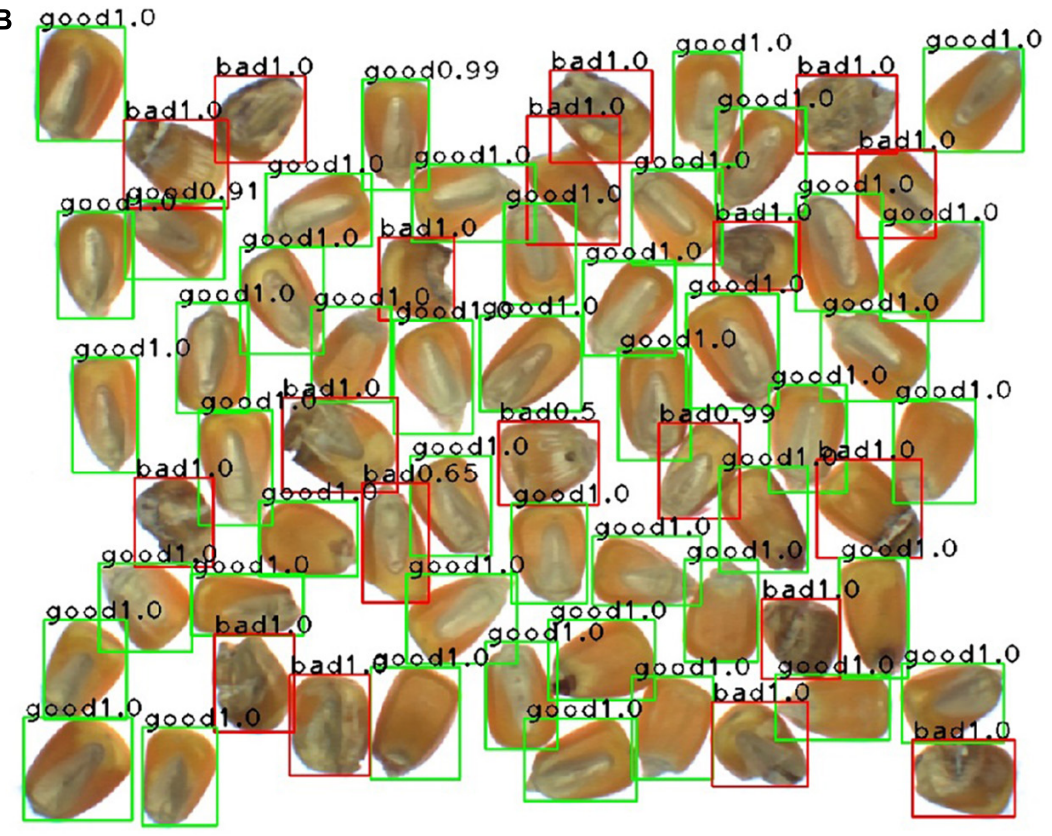

FIGURE 8 | (A) The original image and (B) object detection results.

used in the Xception model, and the accuracy was also relatively low. Considering the accuracy and convergence, VGG16 and ResNet50 were combined to construct the final model.

\section{The Selection of Corn-Seed-Net Model Parameters}

To obtain faster training speed and better convergence performance of the model, the same 4-channel images were used to train the model, and two branches of Corn-seed-Net were initialized with the weights trained using the ImageNet dataset. The influences of different initial learning rates and different optimization algorithms on the model were tested (as shown in Table 1). Figure 7 shows that the SGD algorithm converged faster, and the Adam algorithm was unstable in the first half of the training process. Therefore, the SGD optimization algorithm was used in the experiment, and the initial learning rate was set to 0.001 .

\section{Test Results of the Corn-Seed-Net Model on a Single Seed}

To verify the classification accuracy of the Corn-seed-Net model for 4-channel images of a single seed, 100 corn seeds without any defects in appearance and another 100 seeds with defected appearance were selected in this experiment. At the same time, other one-pathway CNN models were compared, and the results are shown in 
TABLE 3 | Comparison of model performance combined with watershed algorithm.

\begin{tabular}{|c|c|c|c|c|c|c|c|}
\hline Model & Classes & Precision/\% & Recall/\% & Averaged precision/\% & Averaged recall/\% & $\mathbf{F} 1 / \%$ & Detection time/ms \\
\hline \multirow[t]{2}{*}{ VGG16 } & Good & 90.91 & 96.55 & 93.05 & 94.71 & 93.87 & 139.5 \\
\hline & Bad & 95.19 & 92.86 & & & & \\
\hline \multirow[t]{2}{*}{ ResNet50 } & Good & 94.00 & 97.24 & 94.69 & 94.60 & 94.64 & 122.5 \\
\hline & Bad & 95.37 & 91.96 & & & & \\
\hline \multirow[t]{2}{*}{ MobeliNet } & Good & 93.85 & 94.48 & 91.96 & 93.22 & 92.59 & 95.95 \\
\hline & Bad & 91.96 & 91.96 & & & & \\
\hline \multirow[t]{2}{*}{ DenseNet121 } & Good & 91.45 & 95.86 & 92.43 & 92.13 & 92.27 & 136.75 \\
\hline & Bad & 93.40 & 88.39 & & & & \\
\hline \multirow[t]{2}{*}{ Xception } & Good & 94.48 & 94.48 & 93.26 & 93.67 & 93.46 & 133.85 \\
\hline & Bad & 92.03 & 92.86 & & & & \\
\hline \multirow[t]{2}{*}{ Corn-seed-Net } & good & 94.08 & 98.62 & 95.63 & 95.29 & 95.46 & 149.55 \\
\hline & bad & 97.17 & 91.96 & & & & \\
\hline
\end{tabular}

TABLE 4 | Comparison of model performance combined with watershed algorithm using RGB images.

\begin{tabular}{|c|c|c|c|c|c|c|c|}
\hline Model & Classes & Precision/\% & Recall/\% & Averaged precision/\% & Averaged recall/\% & $\mathrm{F} 1 / \%$ & Detection time/ms \\
\hline \multirow[t]{2}{*}{ RGB VGG16 } & Good & 90.13 & 94.48 & 91.67 & 90.10 & 90.87 & 69.92 \\
\hline & Bad & 93.20 & 85.71 & & & & \\
\hline \multirow[t]{2}{*}{ RGB ResNet50 } & Good & 95.56 & 95.86 & 94.54 & 93.02 & 93.77 & 98.83 \\
\hline & Bad & 93.52 & 90.18 & & & & \\
\hline \multirow[t]{2}{*}{ RGB Corn-seed-Net } & Good & 93.33 & 96.55 & 93.80 & 92.47 & 93.13 & 117.63 \\
\hline & Bad & 94.28 & 88.39 & & & & \\
\hline \multirow[t]{2}{*}{ RGB + NIR Corn-seed-Net } & Good & 94.08 & 98.62 & 95.63 & 95.29 & 95.46 & 149.55 \\
\hline & Bad & 97.17 & 91.96 & & & & \\
\hline
\end{tabular}

Table 2. The averaged accuracy of the Corn-seed-Net model for each single seed classification is up to $100 \%$, which was better than other one-pathway models. The averaged detection time for a single seed was $68 \mathrm{~ms}$, which indicated that the two-pathway model is suitable for seed classification.

\section{Detection Results of the Corn-Seed-Net Model Combined With the Watershed Algorithm}

To accurately locate each seed with the quality rating, the watershed algorithm was adopted and combined with the Corn-seed-Net model on 4-channel images of corn seed (Figure 8A). The conglutinated seeds were segmented using the watershed algorithm, and meanwhile, the position coordinates of each seed were also obtained. The detection results are shown in Figure 8B.

To evaluate the performance of this method, 10 groups of images were used for verification. At the same time, other onepathway CNNs were compared, and the results are shown in Table 3. It showed that the watershed algorithm combined with the Corn-seed-Net model had the highest precision and recall rate on average, and the F1 value was $95.46 \%$. Due to the addition of the operation of the watershed segmentation during image detection, there was an increase in the detection time, and the averaged detection time for a single seed was $149.55 \mathrm{~ms}$. The results appeared that the model performance improves when the watershed algorithm was adopted and combined with twopathway CNN.

\section{RGB Images Detection Results}

To investigate whether using 4-channel images (RGB + NIR) is superior to 3-channel images (RGB) in seed classification, RGB images of the same dataset were used in the experiment, with watershed algorithm combined with Corn-seed-Net model, and the results are shown in Table 4. It is shown that the extra information carried with the NIR band improved the model performance on both precision and recall rate, compared with the models obtained with RGB images only.

To fully evaluate the performance of the watershed algorithm combined with the Corn-seed-Net model, the watershed algorithm combined with the traditional feature extraction method was also studied, and SVM was used to classify

TABLE 5 | Comparison of object detection results.

\begin{tabular}{lccc}
\hline Model & Average precision/\% & Average recall/\% & F1/\% \\
\hline GLCM + SVM & 22.05 & 48.11 & 30.24 \\
Color + SVM & 60.97 & 58.74 & 59.83 \\
HOG + SVM & 64.30 & 64.07 & 64.18 \\
MC + SVM & 68.64 & 68.17 & 68.40 \\
LBP + SVM & 74.28 & 73.73 & 74.00 \\
Corn-seed-Net & 95.63 & 95.29 & 95.46
\end{tabular}


the corn seeds based on their quality. The results are shown in Table 5, and it indicates that the precision, recall, and F1 of the method we have proposed were all significantly higher than those of the traditional methods, as more deep image features were extracted in $\mathrm{CNN}$.

\section{DISCUSSION}

At present, some studies have been devised in seed classification using imaging technology combined with machine learning and deep learning (Huang et al., 2019; Kozowski et al., 2019; Ansari et al., 2021). However, most of the studies were based on RGB imaging technology rather than using four-channel multispectral images. Moreover, there are few studies on seed quality detection using the current typical object detection algorithm. This article designed an end-to-end object detection model, and high accuracy was achieved in seed quality detection.

In this article, RGB and NIR images of corn seeds were obtained using a multispectral camera, and the watershed algorithm combined with the Corn-seed-Net model was used to predict the quality of corn seeds. The watershed algorithm is used to segment every single seed and obtain the precise location of the seed. At the same time, while the 4-channel image data with both RGB and NIR bands were used as the inputs of the Corn-seedNet model, the accuracy of the model was better than that with RGB images only.

The Corn-seed-Net model combines the advantages of VGG16 and ResNet50, and deeper information could be extracted by deep networks. It employs a residual network structure, and the effect of degradation of the deep network is eliminated. With the optimized model, 200 single corn seeds were used for verification and compared with other single-pathway models, and the results revealed that the average classification accuracy of the Corn-seed-Net model reached $100.00 \%$.

To evaluate the corn seed defect detection performance of the watershed algorithm combined with the Corn-seed-Net model, we compared the detection results with RGB images and traditional feature extraction methods. The experimental results showed that the proposed method in this article had the best performance, with an average precision of $95.63 \%$, an average recall rate of $95.29 \%$, and an F1 value of $95.46 \%$.

\section{REFERENCES}

Afonso, M., Blok, P. M., Polder, G., Wolf, J., and Kamp, J. (2019). Blackleg detection in potato plants using convolutional neural networks. IFAC PapersOnLine 52, 6-11. doi: 10.1016/j.ifacol.2019.12.481

Afzal, I., Bakhtavar, M. A., Ishfaq, M., Sagheer, M., Baributsa, D., et al. (2017). Maintaining dryness during storage contributes to higher maize seed quality. J. Stored Prod. Res. 72, 49-53. doi: 10.1016/j.jspr.2017.04. 001

Ali, A., Qadri, S., Mashwani, W. K., and Belhaouari, S. B. (2020). Machine learning approach for the classification of corn seed using hybrid features. Int. J. Food Prop. 23, 1097-1111. doi: 10.1080/10942912.2020.1778724

Altuntaş, Y., Cömert, Z., and Kocamaz, A. F. (2019). Identification of haploid and diploid maize seeds using convolutional neural networks and a transfer learning approach. Comput. Electron. Agric. 163:104874. doi: 10.1016/j.compag. 2019.104874

\section{CONCLUSION}

In this study, an end-to-end corn seed object detection model was proposed, which combined watershed segmentation algorithm and CNNs. In comparison with mainstream object detection models (e.g., Faster-RCNN, SSD, and YOLO), our method uses a watershed segmentation algorithm to obtain more accurate target positions, which also reduces the complexity of the network at the same time. In addition, this method eliminates the manual annotation of the image and reduces the workload of dataset preparation. In the future, this method can be further optimized by simplifying the network structure, which may shorten the calculation time while ensuring the classification accuracy, to provide a basis for the subsequent development of a quality detection device.

\section{DATA AVAILABILITY STATEMENT}

The raw data supporting the conclusions of this article will be made available by the authors, without undue reservation.

\section{AUTHOR CONTRIBUTIONS}

LW, JL, and XF conceived the idea, proposed the method, and revised the manuscript. LW, JW, and JZ contributed to the preparation of equipment and acquisition of data, wrote the code, and tested the method. LW, JZ, and JL contributed to the validation results. LW and XF wrote the manuscript. All authors read and approved the final manuscript.

\section{FUNDING}

This study was supported by the National Natural Science Foundation of China (32072572), the Hebei Talent Support Foundation (E2019100006), the Key Research and Development Program of Hebei Province (20327403D), the Talent Recruiting Program of Hebei Agricultural University (YJ201847), and the special project of talent research introduced by Hebei Agricultural University (YJ2020064).

Ansari, N., Ratri, S. S., Jahan, A., Ashik-E-Rabbani, M., and Rahman, A. (2021). Inspection of paddy seed varietal purity using machine vision and multivariate analysis. J. Agric. Food Res. 3:100109. doi: 10.1016/j.jafr.2021.100109

Arunachalam, A., and Andreasson, H. (2021). Real-time plant phenomics under robotic farming setup: a vision-based platform for complex plant phenotyping tasks. Comput. Electrical Eng. 92:107098. doi: 10.1016/j.compeleceng.2021. 107098

Azimi, S., Kaur, T., and Gandhi, T. K. (2020). A deep learning approach to measure stress level in plants due to nitrogen deficiency. Measurement 173:108650. doi: 10.1016/j.measurement.2020.108650

Chollet, F. (2017). Xception: Deep Learning with Depthwise Separable Convolutions. 2017 IEEE Conference on Computer Vision and Pattern Recognition (CVPR). Honolulu: IEEE.

Dalal, N. (2005). "Histograms of oriented gradients for human detection," in 2005 IEEE Computer Society Conference on Computer Vision and Pattern Recognition (CVPR'05), (San Diego, CA: IEEE), 886-893. 
Gao, F., Fu, L., Zhang, X., Majeed, Y., and Zhang, Q. (2020). Multi-class fruit-onplant detection for apple in snap system using faster r-cnn. Comput. Electron. Agric. 176:105634. doi: 10.1016/j.compag.2020.105634

Gutiérrez, S., Wendel, A., and Underwood, J. (2019). Spectral filter design based on in-field hyperspectral imaging and machine learning for mango ripeness estimation. Comput. Electron. Agricu. 164:104890. doi: 10.1016/j.compag.2019. 104890

Haralick, R., Shanmugam, K., and Dinstein, I. (1973). Textural Features for Image Classification, IEEE Trans. on Systems, Man and Cybernetics. SMC 3, 610-621. doi: 10.1109/tsmc.1973.4309314

He, K., Zhang, X., Ren, S., and Sun, J. (2016). “Deep Residual Learning for Image Recognition, in IEEE Conference on Computer Vision and Pattern Recognition (CVPR), (Las Vegas, NV: IEEE).

Huang, G., Liu, Z., Laurens, V., and Weinberger, K. Q. (2016). Densely Connected Convolutional Networks. Honolulu, HI: IEEE Computer Society.

Huang, S., Fan, X., Sun, L., Shen, Y., and Suo, X. (2019). Research on classification method of maize seed defect based on machine vision. J. Sens. 2019, 11-25. doi: 10.1155/2019/2716975

Keiichi, M., Satoru, K., and Komaki, I. (2019). Computer vision-based phenotyping for improvement of plant productivity: a machine learning perspective. GigaScience 8:giy153. doi: 10.1093/gigascience/giy153

Ke-Ling, T. U., Lin-Juan, L. I., Yang, L. M., Wang, J. H., and Sun, Q. (2018). Selection for high quality pepper seeds by machine vision and classifiers. J. Integr. Agric. 17, 1999-2006. doi: 10.1016/s2095-3119(18)62031-3

Kiratiratanapruk, K., and Sinthupinyo, W. (2012). "Color and Texture for Corn Seed Classification by Machine Vision," in International Symposium on Intelligent Signal Processing and Communications Systems, (Chiang Mai: IEEE), doi: 10.1109/ISPACS.2011.6146100

Kozowski, M., Górecki, P., and Szczypiński, P. M. (2019). Varietal classification of barley by convolutional neural networks. Biosyst. Eng. 184, 155-165.

Kusumaningrum, D., Lee, H., Lohumi, S., Mo, C., Kim, M. S., and Kwan Cho, B. (2018). Non-destructive technique for determining the viability of soybean (glycine max) seeds using ft-nir spectroscopy. J. Sci. Food Agric. 98, 1734-1742. doi: 10.1002/jsfa.8646

Laabassi, K., Belarbi, M. A., Mahmoudi, S., Mahmoudi, S. A., and Ferhat, K. (2021). Wheat varieties identification based on a deep learning approach. J. Saudi. Soc. Agric. Sci. 20, 281-289. doi: 10.1016/j.jssas.2021.02.008

Lei, T., Jia, X., Liu, T., Liu, S., Meng, H., and Nandi, A. K. (2019). Adaptive morphological reconstruction for seeded image segmentation. IEEE Trans. Image Process. 28, 5510-5523. doi: 10.1109/TIP.2019.292 0514

Ojala, T., Pietikainen, M., and Maenpaa, T. (2002). "Multiresolution Gray-Scale and Rotation Invariant Texture Classification with Local Binary Patterns," in IEEE Transactions on Pattern Analysis and Machine Intelligence, (Chiang Mai: IEEE), 971-987. doi: 10.1109/tpami.2002.1017623

Pang, L., Men, S., Yan, L., and Xiao, J. (2020). Rapid vitality estimation and prediction of corn seeds based on spectra and images using deep learning and hyperspectral imaging techniques. IEEE Access. 99, 1-1. doi: 10.1109/ACCESS. 2020.3006495
Rehman, T. U., Mahmud, M. S., Chang, Y. K., Jin, J., and Shin, J. (2018). Current and future applications of statistical machine learning algorithms for agricultural machine vision systems. Comput. Electron. Agric. 156, 585-605. doi: 10.1016/j.compag.2018.12.006

Samik, B., and Sukhendu, D. (2018). Mutual variation of information on transfercnn for face recognition with degraded probe samples. Neurocomputing.310, 299-315. doi: 10.1016/j.neucom.2018.05.038

Simonyan, K., and Zisserman, A. (2015). "Very Deep Convolutional Networks for Large-Scale Image Recognition," in Published as a conference paper at ICLR 2015, (Chiang Mai: IEEE). doi: 10.3390/s21082852

Sj, A., Shma, B., Fjv, A., and Am, C. (2021). Computer-vision classification of corn seed varieties using deep convolutional neural network. J. Stored Prod. Res. 92:101800. doi: 10.1016/j.jspr.2021.101800

Sta, B., Xu, M. B., Zm, A., Long, Q. B., and Yw, B. (2019). Segmentation and counting algorithm for touching hybrid rice grains. Comput. Electron. Agric. 162, 493-504. doi: 10.1016/j.compag.2019.04.030

Tiwari, V., Joshi, R. C., and Dutta, M. K. (2021). Dense convolutional neural networks based multiclass plant disease detection and classification using leaf images. Ecol. Inform. 63:101289. doi: 10.1016/j.ecoinf.2021.101289

Zhang, C., Zhao, Y., Yan, T., Bai, X., and Liu, F. (2020). Application of near-infrared hyperspectral imaging for variety identification of coated maize kernels with deep learning. Infrared Phys. Technol. 111:103550. doi: 10.1016/j.infrared.2020. 103550

Zhang, J. J., Ma, Q., Cui, X., Guo, H., and Zhu, D. H. (2020). High-throughput corn ear screening method based on two-pathway convolutional neural network. Comput. Electron. Agric. 175:105525. doi: 10.1016/j.compag.2020.105525

Zhang, L., Li, N., and Fang, H. (2020). Morphological Characteristics and Seed Physiochemical Properties of Two Giant Embryo Mutants in Rice. Rice Sci. 27, 81-85. doi: 10.1016/j.rsci.2019.04.006

Zhang, L., Zou, L., Wu, C., Jia, J., and Chen, J. (2021). Method of famous tea sprout identification and segmentation based on improved watershed algorithm. Comput. Electron. Agric. 184:106108. doi: 10.1016/j.compag.2021.106108

Conflict of Interest: The authors declare that the research was conducted in the absence of any commercial or financial relationships that could be construed as a potential conflict of interest.

Publisher's Note: All claims expressed in this article are solely those of the authors and do not necessarily represent those of their affiliated organizations, or those of the publisher, the editors and the reviewers. Any product that may be evaluated in this article, or claim that may be made by its manufacturer, is not guaranteed or endorsed by the publisher.

Copyright (c) 2022 Wang, Liu, Zhang, Wang and Fan. This is an open-access article distributed under the terms of the Creative Commons Attribution License (CC BY). The use, distribution or reproduction in other forums is permitted, provided the original author(s) and the copyright owner(s) are credited and that the original publication in this journal is cited, in accordance with accepted academic practice. No use, distribution or reproduction is permitted which does not comply with these terms. 